\title{
Physical Assessment Techniques Performed by Jordanian Registered Nurses (RNs): Survey Study
}

\author{
Ola Ahmad Kutah \\ Faculty of Nursing, Isra University, Amman, Jordan
}

\section{Email address:}

ola_kutah@hotmail.com

\section{To cite this article:}

Ola Ahmad Kutah. Physical Assessment Techniques Performed by Jordanian Registered Nurses (RNs): Survey Study. American Journal of Nursing Science. Vol. 10, No. 1, 2021, pp. 41-48. doi: 10.11648/j.ajns.20211001.18

Received: January 12, 2021; Accepted: January 19, 2021; Published: January 28, 2021

\begin{abstract}
Within the nursing curriculum, there is a growing concern about unnecessary content. Since content overload is a growing issue, the content of undergraduate nursing curriculum must be evaluated rigorously as reflected in the nursing literature. In the creation of quality client care in nursing practice, recognizing challenges to performing physical examination techniques among nurses produces a more thorough assessment. This research aimed to investigate the physical assessment techniques performed by Jordanian RNs practicing in both selected public and privet health sectors to gain better understanding of the skills required by graduates of nursing programs in Jordan. A descriptive research design of 138 Jordanian registered nurses working in different practice units using questionnaire was used. The Jordanian registered nurses reported the frequency in which they performed 30 physical assessment techniques. Different statistical methods utilized to analyze the data collected. The results indicated that the Jordanian registered nurses' performance frequency of the selected physical assessment techniques was high. However, there were statistical differences evident regarding the type of healthcare sector, Gender \& work experience variables. The results were in favor in registered nurses working in the public sector, females $\&$ registered nurses with less than ten years of experience. However, there were no statistical differences evident regarding the practice unite variable. A registered nurse must understand how to execute a broad range of techniques to carry out a thorough physical assessment, however the complexity of assessment is not inevitably characterized by the specific techniques applied. And if several of these physical assessment techniques are seldom carried out by registered nurses beyond their specific practice unit, the question arises whether it is suitable to incorporate them in the nursing curriculum.
\end{abstract}

Keywords: Physical Assessment Techniques, Nurses, Education

\section{Introduction}

The adequacy of educational preparation of nursing graduate; and the tremendous content within nursing curricula continue to represent a dilemma [1]. Graduates of nursing programs necessities' various skill base and they should have the ability to work within various clinical settings. Nevertheless, within the nursing profession there is a continuing concern regarding the insufficient preparedness of nursing graduates to enter practice [2-4].

Physical assessment course is a requirement for all university nursing students, regardless of the program length or location of the institution [5]. To this end, the Global Nursing Program has developed a curriculum that is consistent with the physical assessment techniques taught in the program [5]. The problem facing nursing schools is to decide which physical assessment techniques entry-level nurses need and which skills should be mastered "on the job" [6].

Traditionally, performing physical assessments and obtaining health histories from clients in many regions is usually regarded as a medical role instead of a nursing role [7] Health assessment, on the other hand, gives nurses a starting point for developing a nursing care plan. They are able to build a therapeutic rapport with their clients and provide comprehensive care as a result $[8,9]$. Thus, the capacity of a nurse to detect both healthy and pathological physical observations increase their confidence and improves the health outcomes of their clients [10, 11].

Changes in health care field pressure nurses to become dynamic and more competent than any time in recent memory, bridging the gap between nursing service prospects and the real aptitude base of standard nursing graduates. 
Worry about satisfactory readiness of graduates is not exceptional to nursing though.

This research enables us to reflect on education based on findings in practice. It aims to explore the physical assessment techniques most frequently used by Jordanian registered nurses and reflect the findings on the Jordanian nursing curricula.

\subsection{Literature Review}

Physical assessment is a crucial skill for nurses [12], since it is an essential component of the nursing process's first stage, provides a framework for promoting wellness, and provides a shared ground for communication with other health professionals [13].

It is frequently a prerequisite for graduate study and is a foundational skill for students moving into advanced practice areas [12].

Hence, there is sound theoretical bases for incorporating physical assessment techniques in a nursing undergraduate program [13].

However, multiple research studies on physical assessment indicate that only about one-third of the techniques taught in basic nurse education courses are routinely utilized in practice [2, 14-16]. Physical assessment involving the respiratory system were identified as one of the fundamental approaches [15]. It was also established that the core techniques of nurses' physical assessment mainly included vital signs [17].

One would expect that well-defined and specific subject such as physical health assessment rarely raises questions about what to teach. Nonetheless, there is dispute about the extent of physical assessment techniques that must be introduced in undergraduate nursing curricula; this issue is mostly resolved by evidence of what nursing students have learned and performed [1].

Ever Since Giddens [15] published her research findings, this situation has been debatable. The research results emphasized on the physical assessment techniques used by RNs in their workplace [15]. It shed the light on the necessity to scrutinize the scope of physical assessment techniques taught in nursing education since these techniques may not necessarily correspond to the practice area of registered nurses or nursing students $[1,2,8,15,18]$.

A review of recent research on physical assessment in undergraduate nursing curriculum indicates that most procedures taught to nurses in pre-service programs are not applied in clinical settings [1, 2, 19]. Merely subsection of obtained physical assessment techniques has been used by RNs [2, 14, 19].

The results of these studies indicate that there may be a gap between what is taught, and the actual content needed to begin nursing practice and this has been further affirmed by the findings of Giddens \& Eddy study in 2009 [20]. Consequently, it appears more suitable to delineate the physical assessment techniques performed by the RN in clinical practice to more precisely focus on the assessment techniques required for entry-level practice.
Physical health assessment material encompassed in physical textbooks has been continuously expanding to meet health needs. Hence, Giddens \& Eddy [20] argue that the way students and nurses utilize textbooks should be taken into account. They claim that several teachers, especially novice teachers, might be obliged to address all physical assessment techniques described in textbooks [20]. Due to their lack of experience, it is often difficult for students to distinguish important learning techniques from those not related to entry-level practice. This makes several students swamped with a substantial amount of study material which they are unable to learn [20].

All in all physical assessment is an integral aspect of almost all clinical experiences, providing knowledge vital to the client's care and management [21].

However, there were few studies dealing specifically with the amount of utilization of physical assessment techniques following graduation, especially in Jordan.

\subsection{Statement of Problem}

In Jordan physical assessment techniques are taught usually as part of the undergraduate Bachelor of Nursing degree program. Graduates of nursing programs necessities various skill base and they should have the ability to work within various clinical settings. Nevertheless, within the nursing profession there is a continuing dilemma regarding the overwhelming physical health assessment content in the nursing curricula in one hand and the preparedness of nursing graduates to enter practice in the other. Hence, determining the depth to which undergraduate nursing education should include physical assessment topics is essential to ascertain how confident Jordanian RNs are in carrying out physical exams and how often they do it.

To address Jordanian RNs' current knowledge of physical health assessment and the frequency they apply its techniques in their practice, the decision to perform this study was made.

\subsection{Research Questions}

What are the physical assessment techniques performed by Jordanian RNs?

Is there a difference in performing these techniques among Jordanian RNs working in public vs privet hospitals?

Is there a difference in performing these techniques among male vs female RNs?

Is there a difference in performing these techniques according to the years of experience?

Is there a difference in performing these techniques among Jordanian RNs working in different practice units?

\subsection{Justification of Study}

This study's objective was to determine physical assessment techniques performed by practicing Jordanian RNs to achieve better comprehension of the competencies needed by graduates of nursing programs. Determining the frequency of RNs performing specific physical assessment 
techniques and employ the results in the nursing curricula will taper their scope of focus teaching to focus on much needed nursing techniques and practices. This study derives its importance through its scientific addition, which can be used to raise the competencies required for nursing graduates by developing the study plan in nursing faculties and providing them with the necessary skills that qualify them for safe practice when practicing their work.

\section{Methodology}

A descriptive approach has been conducted using a Likert survey questionnaire due to its compatibility to the objectives of this study.

\section{Procedure \& Method}

Prior to data collection, participants were told about the study and ensured of their confidentiality. Data were collected in November 2019. The survey does not contain a personal identification code, but is directly handled and collected by the researcher, which prevents other individuals (such as nurse managers) from observing the individually filled out forms.

\section{Participants \& Sampling}

Using stratified simple random sampling, 138 individuals from units providing direct patient care in inpatient settings from four big public and private health care institutions in Amman who consented to participate in the research were selected. Nursing supervisors, nurse administrators, managers, educators, and advanced practice nurses were not included in the sample (i.e., clinical nurse specialists, nurse practitioners, nurse-midwives, nurse anaesthetists).

\section{Measurement}

The purpose of this research was to assess Jordanian RNs performance of physical assessment techniques in their clinical settings. The researcher created the questioner for this investigation. Following a study of the literature, a collection of 30 regularly used 'core physical assessment techniques' described by Giddens in 2007 [15] were chosen to develop preliminary instrument items.

\subsection{Instrument Validity}

To validate the study questionnaire and to ensure its suitability to the Jordanian culture and to make sure of its usability, it was presented to a group of experts (clinical experts \& lecturers).

Experts assessed the questionnaire's content validity index. They were tasked with evaluating the items' relevance to the objectives and providing feedback on the questionnaire. The experts determined the suitability of the questionnaire for the purpose of this study.

Each questionnaire item was assessed for relevance on a score of 1 4 (1=not relevant, $2=$ somewhat relevant, $3=$ quite relevant, and $4=$ very relevant). Hence, all the 30 core physical assessment techniques were incorporated in the questionnaire as content-validated questions.

\subsection{Instrument Reliability}

Internal consistency and test-retest reliability were used to assess the instrument's reliability. Cronbach's alpha coefficient was computed for the instrument using SPSS, and test-retest reliability was determined over a two-week period with a sample size of 20 individuals (who were excluded from the study).

The reliability coefficient according to Cronbach's alpha was 0.85 while it was 0.89 according to test-retest reliability which were considered acceptable for the applicability of the study.

The data was gathered via the use of a 30-item Likert scale survey on physical assessment techniques. The following 5-point scale was used to gauge the frequency with which participants conducted the physical assessment techniques:

$0=\mathrm{I}$ do not know how to do this technique.

$1=\mathrm{I}$ know how to do this technique, but it is not part of my clinical practice.

2 = I perform this technique rarely (a few times during my career).

$3=\mathrm{I}$ perform this technique frequently in my clinical practice (every 2 to 5 times I work).

4 = I perform this technique regularly in my clinical practice (every time I work).

Results classification:

To characterize the arithmetic average values (low, medium, and high), the findings were translated into the triple rating system presented in Table 1:

Table 1 The classification scale to describe the mean values.

\begin{tabular}{ll}
\hline Value & Description \\
\hline $0-1.33$ & Low \\
$1.34-2.66$ & Medium \\
$2.67-4$ & High \\
\hline
\end{tabular}

The following equation was used to determine the preceding classifications:

$$
\text { Class Width }=\text { Range }\left(\begin{array}{c}
\text { Maximum }- \text { Minimum }) / \text { Number of } \\
\text { classes }
\end{array}\right.
$$

Class Width $=(5-1) / 3=1.33$

\subsection{Return Rates}

Among the 150 surveys circulated, a total of 138 surveys have been completed and returned, with a total return rate of $90 \%$.

\section{Limitations}

While the study's results are intriguing, further research is essential to contextualize them in the framework of educational practice and learning. Longitudinal research that follows people from their educational program to 
professional practice and focuses on the application of physical health assessment techniques might be very intriguing.

The study's participants were recruited using a convenience sample approach, which restricts the generalizability of the study's findings. Hence, this study should be repeated with a wider range of $\mathrm{RN}$ samples from different geographic regions and different practical environments. The main physical health assessment techniques practiced by nurses in various clinical environments must be examined, and the differences in techniques performed by nurses should be compared based on educational background or years of experience. The deliberative process for deciding which techniques should be used for the physical assessment should also be studied.

\section{Demographic Data}

To establish whether participants were a homogeneous or diverse group of Jordanian RNs in relation to numerous criteria, the questions in section one of the questionnaire collected socio demographic data from participants. Health care sector, gender, work experience as an $\mathrm{RN}$, and practice unit (Maternal-infant, Adult, Pediatric, Perioperative) are all included in Table (6.1) below.

As a result of their specific interest in or belief that they might make a substantial contribution to this area, the Jordanian RNs who decided to take part in the research did so voluntarily. There was a wide range of backgrounds, practice units, ages, and years of experience among those who volunteered.

Table 2. The demographics.

\begin{tabular}{|c|c|c|c|}
\hline Characteristics & Variables & Number & Percentage \\
\hline \multirow{2}{*}{ Healthcare Sector } & Privet & 65 & $47 \%$ \\
\hline & Public & 73 & $53 \%$ \\
\hline \multirow{2}{*}{ Gender } & Male & 52 & $38 \%$ \\
\hline & Female & 86 & $62 \%$ \\
\hline \multirow{2}{*}{ Work experience (year) } & $\leq 10$ & 95 & $69 \%$ \\
\hline & $>10$ & 43 & $31 \%$ \\
\hline \multirow{3}{*}{ Practice Unite } & Maternal-infant & 30 & $22 \%$ \\
\hline & Pediatric (i.e., general inpatient pediatrics, pediatric critical care, outpatient pediatrics) & 41 & $30 \%$ \\
\hline & Perioperative & 29 & $21 \%$ \\
\hline Total & & 138 & $100 \%$ \\
\hline
\end{tabular}

There were $62 \%$ female participants and $38 \%$ male participants in this study. $53 \%$ of the participants worked in privet healthcare sector while $47 \%$ of subjects worked in public healthcare sector. $30 \%$ of participants are working in pediatric units, $27 \%$ working in internal medicine, $22 \%$ in Maternal-infant and 21\% surgical unit. About $69 \%$ had less than or equal 10 years of work experience though $31 \%$ of them had more than 10 years of work experience (Table 2).

\section{Results}

This study aimed at evaluating physical assessment techniques among registered nurses in Jordan. This section will present the study results and their interpretation according to the sequence of its questions.

Results related to the first question:

What are the physical assessment techniques performed by nurses?

To answer this question, the means, and standard deviations of the study questionnaire items were calculated for the responses of the study sample individuals to the paragraphs. Table 3 shows the mean values and standard deviations of the physical assessment techniques paragraphs in descending order.

Table 3. Mean values \& standard deviations of the physical assessment techniques paragraphs.

\begin{tabular}{|c|c|c|c|c|c|}
\hline Rank & Physical Assessment Technique & Mean & Standard deviation & Percentage $\%$ & Rate \\
\hline 1 & Inspect overall skin color & 3.90 & 0.72 & 98 & high \\
\hline 2 & Evaluate breathing effort & 3.89 & 0.68 & 97 & high \\
\hline 3 & Inspect extremities for skin color and hair growth & 3.89 & 0.68 & 97 & high \\
\hline 4 & Inspect and palpate extremities for oedema & 3.89 & 0.65 & 97 & high \\
\hline 5 & Palpate extremities for temperature & 3.85 & 0.73 & 96 & high \\
\hline 6 & Palpate and inspect capillary refill & 3.81 & 0.72 & 95 & high \\
\hline 7 & Palpate distal pulses for circulation & 3.81 & 0.72 & 95 & high \\
\hline 9 & Inspect wounds & 3.79 & 0.78 & 95 & high \\
\hline 10 & Inspect skin lesions & 3.77 & 0.72 & 94 & high \\
\hline 11 & Inspect external eyes & 3.70 & 0.72 & 93 & high \\
\hline 12 & Evaluate speech & 3.68 & 0.68 & 92 & high \\
\hline 13 & Evaluate face for movement and sensation & 3.66 & 0.68 & 92 & high \\
\hline 14 & Assess hearing based on conversation & 3.22 & 0.65 & 81 & high \\
\hline 15 & Inspect chest shape & 3.21 & 0.73 & 80 & high \\
\hline
\end{tabular}




\begin{tabular}{|c|c|c|c|c|c|}
\hline Rank & Physical Assessment Technique & Mean & Standard deviation & Percentage $\%$ & Rate \\
\hline 17 & Palpate abdomen for tenderness and distension & 3.21 & 0.68 & 80 & high \\
\hline 18 & Inspect the oral cavity & 3.17 & 0.68 & 79 & high \\
\hline 19 & Assess using Glasgow Coma Scale & 3.13 & 0.65 & 78 & high \\
\hline 20 & Assess mental status and level of consciousness & 3.10 & 0.73 & 78 & high \\
\hline 21 & Assess gait & 3.03 & 0.72 & 76 & high \\
\hline 22 & Inspect muscles and extremities for size and symmetry & 2.92 & 0.81 & 73 & high \\
\hline 23 & Observe range of motion of joints & 2.81 & 0.74 & 70 & high \\
\hline 25 & Inspect and examine stool & 2.64 & 0.72 & 66 & Medium \\
\hline 26 & Inspect the spine & 2.61 & 0.66 & 65 & Medium \\
\hline 27 & Auscultate lung sounds & 2.60 & 0.79 & 65 & Medium \\
\hline 28 & Auscultate abdomen for bowel sounds & 2.55 & 0.76 & 64 & Medium \\
\hline 29 & Auscultate heart sounds & 2.53 & 0.62 & 63 & Medium \\
\hline 30 & Assess for PERRLA (Pupils equal, round, reactive to light and accommodation) & 2.52 & 0.56 & 63 & Medium \\
\hline Total & & 3.37 & 0.56 & 84 & High \\
\hline
\end{tabular}

Note. PERRLA=Pupils equal, round, reactive to light and accommodation.

Table 3 shows the mean values and standard deviations for participants' responses to the questionnaire items related to the physical assessment techniques. It is evident that the physical assessment techniques rank was high as the total estimated mean value was (3.37) with a percentage of $84 \%$. The mean values ranged between $(2.52$ - 3.90). Item No. (1) "Inspect overall skin color" achieved the first rank which is considered high with a mean value of (3.90) \& a percentage of 98\%, while Item No. (30) "Assess for PERRLA (Pupils equal, round, reactive to light and accommodation)" came last which is considered medium with a mean value of (2.52) $\&$ a percentage of $63 \%$. In total the number of items that achieved high rank was 24 while the remain 6 achieved medium ranks the thing that indicate a high frequency of Jordanian RNs performance regarding the physical assessment techniques included in the questionnaire.

Results related to the second question:

Is there a difference of performing physical assessment techniques among nurses according to the type of healthcare sector?

To answer this question, the means, and standard deviations of the responses to the questionnaire of the study were calculated. t.Test analysis was used to compare between health assessment techniques carried out by Jordanian RNs according to the healthcare sector variable (private vs public). Table 4 displays that.

Table 4. The results of the t-test for the mean differences of the study sample responses to the questionnaire items according to the healthcare sector variable (private vs public).

\begin{tabular}{lllll}
\hline Healthcare sector & N & Mean & Standard Deviation & t-value \\
\hline Privet & 65 & 3.68 & 0.54 & Sig \\
Public & 73 & 4.02 & 0.61 & $\mathbf{3 . 1 1}$ \\
\hline
\end{tabular}

Table 4 shows that there are significant differences at the level of $(\alpha=0.05)$ between the mean values of the study sample responses to the questionnaire items in terms of the healthcare sector variable (private vs public), and this is based on the calculated t-value of (3.11), as well as the level of significance $(0.001)$ for the total score of the questionnaire, hence, these results considered statistically significant and in favor of the study sample RNs working in public hospitals based on the mean value of (4.02) in comparison with the mean value of (3.68) for the RNs working in the privet sector.

Results related to the third question:

Is there a difference in performing physical assessment techniques among nurses according to the gender?

To answer this question, the means, and standard deviations of the responses to the study questionnaire were calculated t.Test analysis was used to compare between the health assessment techniques carried out by nurses according to the gender variable (male vs female). Table 5 shows that.

Table 5. The results of the t-test for the mean differences of the study sample responses to the questionnaire items according to the gender variable (male vs female).

\begin{tabular}{llllll}
\hline Gender & N & Mean & Standard Deviation & t-value & Sig \\
\hline Male & 52 & 4.24 & 0.59 & \multirow{2}{2}{$\mathbf{2 . 7 3}$} & $\mathbf{0 . 0 0 7}$ \\
Female & 86 & 4.54 & 0.48 & \\
\hline
\end{tabular}

Table 5 shows that there are significant differences at the level of $(\alpha=0.05)$ between the mean values of the study sample responses to the questionnaire items in terms of the gender variable, based on the calculated t-value of (2.73), and with a significant level of (0.007) for the total score of the questionnaire, hence, these results considered statistically significant and in favor of female RNs based on the mean value of (4.54) which was greater than the mean value of the males RNs that was (4.24).

Results related to the fourth question: 
Is there a difference in performing physical assessment techniques among nurses according to the years of experience?

To answer this question, the means, and standard deviations of the responses to the study questionnaire were calculated. t.Test analysis was utilized to examine the health assessment techniques performed by Jordanian RNs based on their years of experience. Table 6 shows that.

Table 6. The results of the t-test for the mean differences of the study sample responses to the questionnaire items according to the years of experience variable.

\begin{tabular}{lllll}
\hline Years of experience & N & Mean & Standard Deviation & t-value \\
\hline$\leq 10$ years & 95 & 3.41 & 0.51 & Sig \\
$>10$ years & 43 & 3.12 & 0.59 & 3.12 \\
\hline
\end{tabular}

Table 6 shows that there are significant differences at the level of $(\alpha=0.05)$ between the mean values of the study sample responses to the questionnaire items in terms of the years of work experience variable (10 years or less, more than 10 years) based on the calculated value of T (3.12), and with a level of significance $(0.001)$ for the total score of the questionnaire, hence, these results considered statistically significant and in favor of RNs with 10 years or less of experience based on the mean value of (3.41), whereas the mean value for nurses with 10 years or more of experience was (3.12).

Results related to the fifth question:

Is there a difference in performing physical assessment techniques among nurses according to the practice unit?

To answer this question, the means and standard deviations of the responses to the study questionnaire were calculated. t.Test analysis was utilized to evaluate health assessment approaches performed by Jordanian RNs based on the practice unit variable. Table 7 shows that.

Table 7. The results of the t-test for the mean differences of the study sample responses to the questionnaire items according to the practice unit variable.

\begin{tabular}{|c|c|c|c|}
\hline Practice Unit & $\mathbf{N}$ & Mean & Standard Deviation \\
\hline Maternal-infant & 30 & 3.31 & 2.11 \\
\hline Adult (i.e., medical-surgical, adult critical care, adult outpatient) & 38 & 3.26 & 3.56 \\
\hline Pediatric (i.e., general inpatient pediatrics, pediatric critical care, outpatient pediatrics) & 41 & 3.30 & 4.11 \\
\hline Perioperative & 29 & 3.24 & 3.24 \\
\hline Total & 138 & 3.28 & 2.21 \\
\hline
\end{tabular}

To find out whether there were statistically significant differences at the level of $(\alpha=0.05)$ between the means of the study sample responses to the questionnaire items in terms of the practice unit variable (Maternal-Infant, Adults, Paediatrics, Perioperative), One-WAY ANOVA has been used and Table 8 shows it.

Table 8. The results of the One-Way ANOVA analysis in light of the practice unit variable.

\begin{tabular}{lllll}
\hline Source & Degrees of Freedom & Sum of Squares & Mean Square & Sig \\
\hline Between & 3 & 54.15 & 18.05 \\
Within & 134 & 2220.12 & 16.57 \\
Total & 137 & 2274.27 & \\
\hline
\end{tabular}

Table 8 shows the results of one-way analysis of variance, that has been applied to find out whether there are significant differences at the level of $(\alpha=0.05)$ between the mean values of the study sample responses to the questionnaire items in terms of the practice setting variable (Maternal-Infant, Adults, Paediatrics, Perioperative).

The $\mathrm{F}$ value was (1.08), and the level of significance was (2.11) for the total score of the questionnaire, which indicates that this result is not statistically significant, and that the health assessment techniques performed by RNs do not differ according to their practice unit.

\section{Discussion}

These results indicate that although this sample of Jordanian RNs integrates quite small set of physical assessment techniques into their practice, but they perform all of them either on a regular or frequent bases.

These findings seem to corroborate those of three more studies, all of which imply that only a limited number of techniques are regularly used in clinical practice $[14,22]$. One could argue that the quantity and the quality of material taught in nursing programs is suitable, but that RNs are not performing adequate comprehensive assessments on their clients, due to lack of either time or supposed need. Likewise, it is likely that nursing programs are offering of a wide range of techniques far greater than is required in clinical practice. The relationship amongst the range of techniques taught in a nursing programs and the techniques performed by RNs is not tackled in the literature, nor was clarified by this study.

Even Though this study did not relate what nurses learned in nursing school, particularly in the physical assessment course, with what they did in practice, one may argue that the majority of nursing schools teach physical assessment at a much deeper level than the 30 fundamental competencies rated by these participants.

Findings from data analysis indicate that female RNs were performing physical assessment techniques more than male RNs and this might be due to the higher number of female nurses practicing nursing in Jordan as shown in the WHO [23] 
recent reports which indicated that female nurse represent $(60 \%)$ of the nursing workforce whereas male nurses represent only $(40 \%)$. And this could be also rendered to the widespread perception that nursing is a female profession and predominantly occupied by female nurses.

As it shown in the results Jordanian RNs working in the public sector seemed to perform physical assessment techniques far more than the ones working the privet sector and this might be rendered to the fact that RNs working in the public sector are offered far more opportunities to attend educational courses, workshops and training sessions funded totally by the government. It also may be argued that RNs working in privet sector has less autonomy and are not permitted to talk or provide advice or ideas to physicians based on the physical assessment they performed and the nursing diagnoses they articulated.

The results also indicated that Jordanian RNs with less than ten years of experience in the field were performing physical assessment techniques far more than the ones with equal or more than ten years of experience and this may be rendered to the progress in the Jordanian nursing programs in terms of concentrating more on enhancing critical thinking, decision making and competency-based learning as a method of educating graduate. According to Benner [24], years spent on the job in the same or comparable settings helps establish competence, yet it would not provide a complex reflexive thinking aspect of learning which most of the recent Jordanian nursing curriculum are keen to pass it to their newly graduate [25].

Finally, the results showed no difference in performing different physical assessment techniques by Jordanian RNs according to their practice unit. While some physical assessment techniques are exclusive to specific practice units, the techniques assessed by the entire sample embody all or most of the techniques of the included practice units. And this is due to the discrepancies in the assessment techniques performed by RNs in different practice units, given the demands of the health clients served; still, several techniques are used with all health clients in all practice units.

\section{Implications for Nursing Education}

The core of nursing education must mirror tangible nursing practice. The results of this research illuminate the frequency of physical health assessment techniques performed by Jordanian RNs in the practice environment of this sample and might offer an in-depth understanding of the physical health assessment content and the techniques targeted in the nursing curricula. Nurse educators should evaluate the existing anticipations of the physical health assessment techniques taught in their courses and contemplate the logic of these anticipations.

Reducing the content of the physical health assessment techniques taught may be beneficial to nursing students. Nurse educators may want to put away their goniometers, picometers, tuning forks, and ophthalmoscopes, while helping students acquire more techniques to observe and identify subtle and obvious clues that indicate changes in the client's condition.
Simultaneously, nurse educators must avoid overly simple assessment methods. When circumstances permit, nurses need to understand when and how to execute additional assessment techniques as the situation demands.

\section{Conclusion}

This study examined the use of physical health assessment techniques by Jordanian RNs in an environment where nursing responsibilities are constantly changing. The regularity with which these techniques were performed varied throughout the 30-core physical health assessment techniques included in this research.

RNs have a limited amount of time with each client, as well as a limited amount of time to acquire and perfect the skills essential for providing high quality care. Hence, nurses are displaying the physical health assessment techniques that they believe are most applicable to daily nursing practice to improve client prognosis.

Given the saturation of nursing education content and the disparity between the techniques taught and those commonly used in practice, it seems obvious that nurse educators should assess and reassess the physical health assessment content taught in nursing programs, as well as consider revising the curriculum and course expectations.

Assuming that nursing graduates will acquire and master all of the techniques necessary throughout all fields of nursing practice is implausible. Perhaps in this instance, less is more. It is recommended that nurse educators consider teaching fewer techniques and focusing on the areas of physical health assessment that are most pertinent to daily nursing practice in the undergraduate nursing curriculum, with an emphasis on interpreting physical health assessment results and developing clinical judgment. Further techniques can be learned in the specific environment where the practice takes place according to the clinical setting needs.

Graduates who have basic physical assessment techniques and the ability to comprehend interpretation of the physical health assessment findings should be able to learn and apply other health assessment techniques that reflect professional practice.

\section{Conflict of Interest}

The author does not have any conflicts of interest.

\section{Acknowledgements}

"The author received no specific funding for this work."

\section{References}

[1] Douglas, C., Windsor, C. \& Lewis, P. 2015, "Too much knowledge for a nurse? Use of physical assessment by final-semester nursing students", Nursing \& Health Sciences, vol. 17 , no. 4, pp. 492-9. 
[2] Birks, M., Cant, R., James, A., Chung, C. \& Davis, J. 2013, "The use of physical assessment skills by registered nurses in Australia: issues for nursing education", Collegian, vol. 20, no. 1, pp. 27-33.

[3] Douglas, C., Booker, C., Fox, R., Windsor, C., Osborne, S. \& Gardner, G. 2016, "Nursing physical assessment for patient safety in general wards: reaching consensus on core skills", Journal of Clinical Nursing, vol. 25, no. (13-14), pp. $1890-900$.

[4] Fusner, S. M., Moots, H., O'Brien, T. \& Sinnott, L. T. 2020 , "Faculty Perceptions of the Importance of Physical Assessment Skills Taught in Prelicensure Nursing Education", Nurse Educator, vol. 45 , no. 5, pp. 248-251.

[5] Morrell, S., Ralph, J., Giannotti, N., Dayus, D., Dennison, S. \& Bornais, J. 2019, "Physical assessment skills in nursing curricula: a scoping review protocol", JBI Database System Reviews and Implementation Reports, vol. 17, no. 6, pp. 1086-1091.

[6] Kohtz, C., Brown, S. C., Williams, R. \& O'Connor, P. A. 2017, "Physical assessment techniques in nursing education: A replicated study", Journal of Nursing Education, vol. 56, no. 5, pp. $287-91$.

[7] Richardson, A. 2020, "Enhancing physical assessment skills in the preregistration nursing curriculum", British Journal of Cardiac Nursing, vol. 15, pp. 1-3.

[8] Fennessey, A. \& Wittmann-Price, R. A. 2011, "Physical assessment: a continuing need for clarification", Nursing Forum, vol. 46, no. 1, pp. 45-50.

[9] College of Nurses of Ontario 2018, Nursing Education Program Approval. Available: http://www.cno.org/en/become-a-nurse/nursing-educationprogram-approval/ [2021, January/9th].

[10] Buykx, P., Kinsman, L., Cooper, S., McConnell-Henry, T., Cant, R., Endacott, R. \& Scholes, J. 2011, "FIRST (2) ACT: Educating nurses to identify patient deterioration - A theory-based model for best practice simulation education", Nurse Education Today, vol. 31, pp. 687-93.

[11] Tuzer, H., Dinc L. \& Elcin, M. 2016, "The effects of using high-fidelity simulators and standardized patients on the thorax, lung, and cardiac assessment skills of undergraduate nursing students", Nurse Education Today, vol. 45, pp. $120 \pm 125$.

[12] Lashley, M., 2005, "Teaching health assessment in the virtual classroom", Journal of Nursing Education, vol. 44, no. 8, pp. 348-50.
[13] Colwell, C. B. \& Smith, J. 1985, "Determining the use of physical assessment skills in the clinical setting", Journal of Nursing Education, vol. 24, no. 8, pp. 333-9.

[14] Secrest, J. A., Norwood, B. R. \& DuMont, P. M. 2005, "Physical assessment skills: a descriptive study of what is taught and what is practiced", Journal of Professional Nursing, vol. 21, no. 2, pp. 114-8.

[15] Giddens, J. F. 2007, "A survey of physical assessment techniques performed by RNs: lessons for nursing education", Journal of Nursing Education, vol. 46, no. 2, pp. 83 \pm 87 .

[16] Cicolini, G., Tomietto, M., Simonetti, V., Comparcini, D., Flacco, M. E., Carvello, M., Manzoli, L. 2015, "Physical assessment techniques performed by Italian registered nurses: a quantitative survey", Journal of Clinical Nursing, vol. 24, no. $23 \pm 24$, pp. $3700 \pm 37066$.

[17] Osborne, S., Douglas, C., Reid, C., Jones, L., Gardner, G. \& Council RPAR 2015, "The primacy of vital signs--acute care nurses' and midwives' use of physical assessment skills: a cross sectional study", International Journal of Nursing Studies, vol. 52, no. 5, pp. $951 \pm 962$.

[18] Shinozaki, E.\& Yamauchi, T. 2009, "Nursing competencies for physical assessment of the respiratory system in Japan", Nursing \& Health Sciences, vol. 11, pp. 285-92.

[19] Lesa, R. \& Dixon, A. 2007, "Physical assessment: implications for nurse educators and nursing practice", International Nursing Review, vol. 54, no. 2, pp. 166-72.

[20] Giddens, J. F. \& Eddy, L. 2009, "A survey of physical assessment skills taught in undergraduate nursing programs: are we teaching too much?", Journal of Nursing Education, vol. 48 , no. 1 , pp. 24-9.

[21] Yudkowsky, R., Otaki, J., Lowenstein, T., Riddle, J., Nishigori, H. \& Bordage, G. A. 2009, "Hypothesis-driven physical examination learning and assessment procedure for medical students: initial validity evidence", Medical Education, vol. 43, no, 8, pp. 729-40.

[22] Barbarito, C., Carney, L., \& Lynch, A. 1997, "Refining a physical assessment course", Nurse Educator, vol. 22, no. 3, pp. 6.

[23] World health organization 2020, State of the World's Nursing Report 2020. Available: https://www.who.int/publications/i/item/9789240003279 [2021, January/9th].

[24] Benner, P. 1984, From novice to expert: Excellence and power in clinical nursing practice, Menlo Park, CA: Addison-Wesley

[25] Bobay, K. L. 2004, "Does experience really matter? Nursing Science", Quarterly, vol. 17, pp. 313-316. 